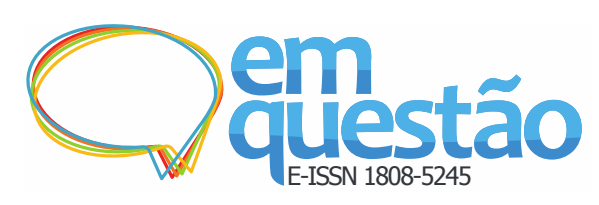

\title{
O estado da blogosfera científica brasileira
}

\author{
Sibele Fausto \\ Bacharel; Universidade de São Paulo, São Paulo, SP, Brasil; \\ sifausto@usp.br \\ Roberto Takata \\ Doutor; Universidade Estadual de Campinas, Campinas, SP, Brasil; \\ rmtakata@gmail.com \\ Nathai Teresa Moreno \\ Graduanda; Universidade de São Paulo, Ribeirão Preto, SP, Brasil; \\ nathaimoreno@gmail.com \\ Alexcolman Tochukwu Apunike \\ Graduando; Universidade de São Paulo, Ribeirão Preto, SP, Brasil; \\ alexcolman.apunike@gmail.com; \\ Jade Lorena Mariano Bucci \\ Graduanda; Universidade de São Paulo, Ribeirão Preto, SP, Brasil; \\ jadebucci@gmail.com \\ Ana Carolina Gonçalves dos Santos \\ Graduanda; Universidade de São Paulo, Ribeirão Preto, SP, Brasil; \\ anacarolinags@usp.br \\ Walas João Ribeiro da Silva \\ Graduando; Universidade de São Paulo, Ribeirão Preto, SP, Brasil; \\ walas.silva@usp.br \\ Mariane Matias \\ Graduanda; Universidade de São Paulo, Ribeirão Preto, SP, Brasil; \\ mariane.matias@usp.br \\ Osame Kinouchi \\ Doutor; Universidade de São Paulo, Ribeirão Preto, SP, Brasil; \\ okinouchi@gmail.com
}

Resumo: Este trabalho investiga quantitativamente a trajetória da blogosfera científica brasileira através de dados estatísticos. A amostra é constituída por 346 blogs científicos do banco de dados Anel de Blogs Científicos, que compreende grande parte dos blogs científicos no Brasil, verificando a atividade destes entre as datas da primeira postagem e a postagem mais recente até novembro de 2015. São analisados dados sobre gêneros de blogueiros, temática científica, distribuição regional e tempo de atividade dos blogs, e curva de crescimento dos blogs ativos. Os resultados mostram que em $37 \%$ dos blogs participam mulheres e em $74 \%$ dos blogs participam homens; a temática predominante é a de Ciências da Vida; a maioria dos blogs se concentra nas regiões Sul e Sudeste do país; e a vida média sobre todos os blogs é de 4,8 anos, com um coeficiente de variação de 0,58. Um achado importante é que o número de blogs ativos parece ter diminuído nos últimos anos. Conjecturamos que esse fenômeno é devido à concorrência com 
outras mídias sociais (Facebook, Twitter e video blogs), entre outras possíveis causas.

Palavras-chave: Blogs científicos. Blogosfera científica brasileira. Mídias sociais. Divulgação científica.

\section{Introdução}

Os blogs surgiram há apenas cerca de 20 anos, desde que o termo weblog foi cunhado por John Barger, em 1997 (DI LUCCIO; NICOLACIDA-COSTA, 2010), mas Mauranen (2013) observa que nesse curto espaço de tempo tais ferramentas de escrita digital se estabeleceram como uma característica permanente do ambiente online. Essas novas ferramentas de comunicação digital proliferaram na década seguinte, percorrendo uma trajetória pontuada por aportes tecnológicos que revolucionaram a forma de comunicação virtual, com os conteúdos disseminados na rede passando a ser gerados pelos próprios usuários (User Generated Content - UGC), interativos, participativos e compartilhados.

As facilidades de operar na rede virtual com as novas ferramentas de publicação digital impulsionaram o surgimento de blogs, criando o ambiente denominado como blogosfera e segmentando os blogs de acordo com seu conteúdo e público-alvo. A agregação de vários blogs por escopo e temáticas comuns representa as várias esferas no universo dos blogs. Segundo Costa (2012, doc. não paginado), “[...] a blogosfera é vista então como um espaço livre e fluido de acúmulo de informações, em uma grande comunidade de troca de conhecimentos".

Aqueles blogs com foco na temática ciência "[...] instituíram-se como uma prática social da comunidade científica e vêm sendo utilizados com diversas finalidades por essa comunidade" (GOMES; FLORES, 2012, p. 394), comunidade esta composta por pesquisadores, jornalistas científicos ou divulgadores em geral (OLIVEIRA, 2011). Verificou-se o surgimento de vários blogs de ciência internacionais, utilizados de forma intensiva mesmo por revistas científicas reconhecidas, como a Nature, a Science, o British Medical Journal (BMJ), entre outras, além da criação de sites coletivos (os chamados 
condomínios de blogs), tais como Research Blogging1, Scienceblogs ${ }^{2}$, ScienceSeeker ${ }^{3}$.

O mesmo fenômeno se apresenta no Brasil, e observa-se uma dispersão desses blogs científicos, com algumas iniciativas pioneiras de agregá-los, como é o caso da plataforma "Roda de Ciência", reunião espontânea de um grupo de blogueiros de ciência surgida em 2006 (GUIMARÃES, 2006) e atualmente desativada; do "Anel de Blogs Científicos" (ABC), projeto da Universidade de São Paulo no campus Ribeirão Preto (USP-RP) lançado em 2008; e, mais recentemente, da plataforma "Blogs de Ciência", da Universidade Estadual de Campinas (Unicamp), lançada em novembro de $2015^{4}$. Também há os correspondentes nacionais de condomínios estrangeiros, como o Research Blogging PT e o Scienceblogs Brasil ${ }^{5}$, reunindo as postagens de blogs de ciência em língua portuguesa.

Tais blogs científicos, percebidos como espaço de interação entre cientistas-blogueiros e leitores, tanto cientistas como não cientistas, inclusive diluindo as fronteiras entre cientistas, comunicadores de ciência e leitores leigos (SHANAHAN, 2011), têm sido objeto de atenção por seu papel na comunicação e na divulgação de informações científicas.

Uma busca na base de dados Scopus ${ }^{\circledR}$ com a expressão "science or scientific and blog or blogging" no título, resumo e/ou nas palavras-chave retorna 1.623 documentos indexados desde 2000, explicitando o grande interesse sobre essa temática na literatura internacional. As várias abordagens vão desde discussões sobre definição, escopo e abrangência dos blogs científicos (WILKINS, 2008); o engajamento de cientistas na blogosfera e as motivações para isso (BATTS; ANTHIS; SMITH, 2008; BIK; GOLDSTEIN, 2013; BONETTA, 2007); as comunidades de blogueiros e suas características e práticas (DENNEN, 2009, 2014; SCHMIDT, 2007); estudos quantitativos explorando a blogosfera científica (FAUSTO et al., 2012); a tentativas de análises sociológicas das práticas da blogosfera científica (JARREAU, 2015), e mesmo sua utilidade na avaliação alternativa da ciência (THELWALL; KOUSHA, 2015).

Já entre os trabalhos nacionais que se propõem a análises dos blogs científicos brasileiros há estudos variados, majoritariamente qualitativos, 
abordando sua estrutura discursiva (análise de discurso) (FLORES, 2016), relações em rede (grafos de links) (CAREGNATO; SOUSA, 2010; SOUSA, 2011), semântica (nuvem de tags e palavras) (AQUINO, 2009; GUIMARÃES, 2008), interação e participação do público leitor (FRANÇA, 2014), etc. Porém, verifica-se uma carência de abordagens quantitativas focando padrões temporais de postagens desses blogs nacionais. Fausto, Takata e Kinouchi (2014) empreenderam um estudo quantitativo preliminar sobre o perfil evolutivo da atividade dos blogs de ciência no contexto nacional, mas sem discutir as causas subjacentes a essa evolução, e sugerindo futuras investigações incluindo outras categorias de análise mais abrangentes, como o local de origem dos blogs e o gênero dos blogueiros, entre outras possíveis para uma visão mais integral da evolução dos blogs científicos no país.

Entendida como um fenômeno da comunicação científica nas novas mídias digitais, a blogosfera científica se presta a uma análise de caráter webométrico, no sentido de um "[...] estudo quantitativo dos aspectos relacionados aos documentos disponibilizados na web." (THELWALL, $2008^{6}$ apud SOUSA, 2011, p. 11). Este trabalho teve o objetivo de verificar o estado da blogosfera científica brasileira para obter um retrato de como esta se apresenta em relação a algumas variáveis, como localização geográfica, gênero dos blogueiros, temáticas e evolução ao longo do tempo, visando contribuir para a compreensão de um importante fenômeno dedicado à divulgação e à popularização da ciência.

\section{Metodologia}

A amostra estudada consiste em 346 blogs listados no Anel de Blogs Científicos $(\mathrm{ABC})$, mantido pelo Laboratório de Divulgação Científica e Cientometria (LDCC), lotado no Departamento de Física da Faculdade de Filosofia, Ciências e Letras da Universidade de São Paulo, campus Ribeirão Preto (FFCL-USP-RP). Essa escolha se justifica pelo fato de o ABC ser a mais antiga fonte de blogs de ciência em língua portuguesa no país, sendo ativa desde 2008, registrando e categorizando os recursos em seu website ${ }^{7}$. 
Todos os blogs investigados são brasileiros e escritos primariamente em português. No site, os blogs estão arrolados por temas (ciências da vida, ciências físicas e astronomia, etc.) e são diretamente acessíveis usando-se os links do ABC. Ao fazer isso, coletamos dados como estado federativo de origem do blog; dados dos blogueiros, permitindo sua definição por gênero; e data do primeiro e último post. Assim, para cada blog foram levantadas as datas da primeira postagem (dia, mês e ano) e da postagem mais recente até a data do levantamento deste estudo (em 5 de novembro de 2015).

O período de atividade do blog corresponde a todo aquele compreendido entre a data da primeira postagem e a data da postagem mais recente, até o levantamento. Foram desconsiderados os blogs que não apresentavam a data completa de postagem (dia/mês/ano) e também aqueles que não possuíam arquivo de postagens em que pudesse ser recuperada a postagem mais antiga.

A partir dessas datas calculamos o tempo de vida de cada blog, permitindo a determinação do tempo de vida médio (separado por temática) e seu coeficiente de variação CV (desvio-padrão dividido pelo tempo de vida médio). As datas de primeiro e último post também nos fornecem uma curva de "nascimento" de blogs $N(t)$ e uma curva de "morte" de blogs $M(t)$. Definimos um blog como "morto" se o blogueiro não publicou nenhum post no último ano. Obtemos assim uma curva de $b \log s$ ativos $A(t)=N(t)-M(t)$.

Os dados assim obtidos foram tabulados e os resultados demonstrados através de gráficos que explicitam a origem geográfica dos $b \log s$, o gênero dos blogueiros, as temáticas e a evolução temporal dos blogs analisados.

\section{Resultados e discussão}

A localização geográfica dos blogs é apresentada na Figura 1 (162 blogs não apresentaram esta informação). Sem muita surpresa, constatamos que a maior parte dos blogs se concentra nas unidades federativas com grande número de universidades, tais como São Paulo, Rio de Janeiro, Minas Gerais e Rio Grande do Sul, todas localizadas nas regiões Sul e Sudeste do país. São Paulo liderou em número de blogs, com 88 ocorrências, perfazendo um quarto $(25,9 \%)$ do total da 
amostra, seguido do Rio de Janeiro, com 34 blogs (10,0\% do total). Minas Gerais

e Rio Grande do Sul disputaram o terceiro lugar em número de blogs (MG=13 blogs, 3,8\% e RS=12 blogs, 3,5\%). Devemos notar também que foram encontrados blogs científicos com informação geográfica em apenas 16 das unidades da federação.

Figura 1 - Distribuição geográfica dos blogs brasileiros (por unidades da federação).

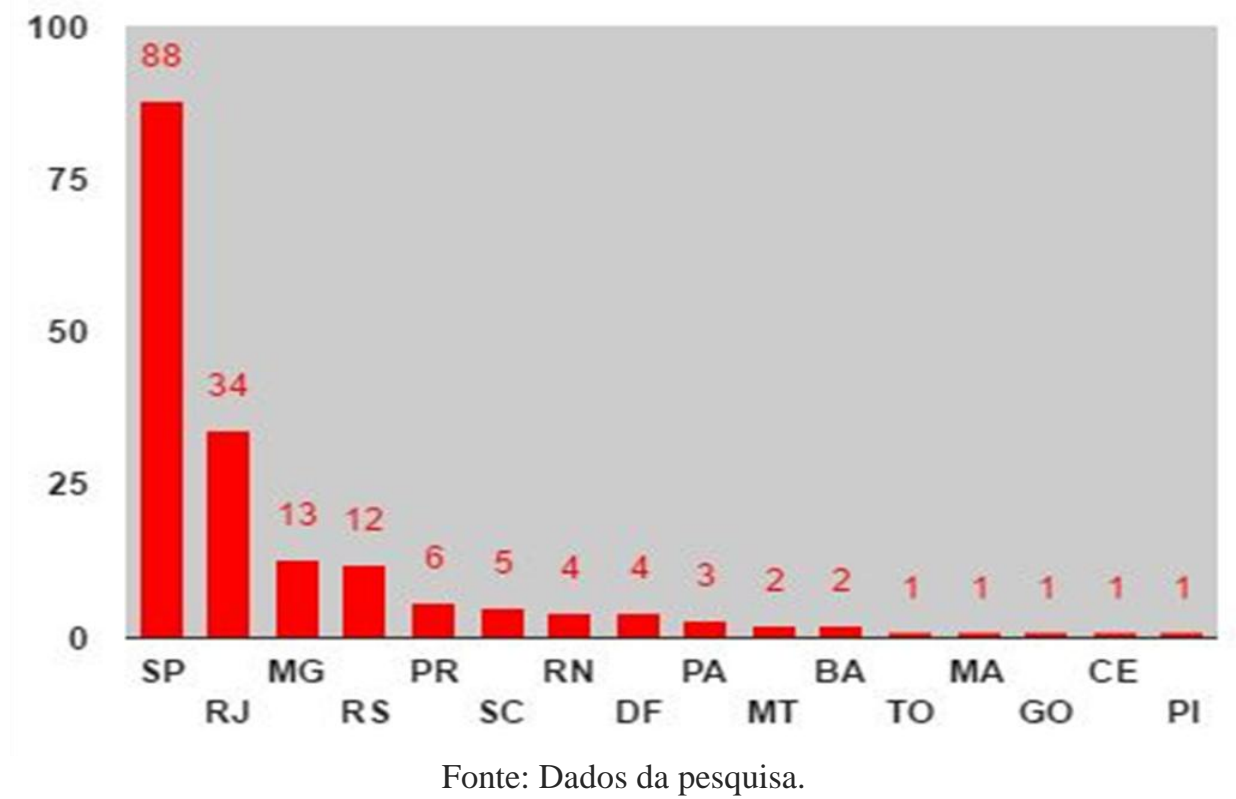

Na Figura 2 apresentamos a estatística de gênero dos blogueiros. O item "ambos" se refere ao fato de que o blog é mantido por pelo menos dois blogueiros de ambos os sexos, isso ocorrendo em $15 \%$ dos blogs. A partir desses dados vemos que em $22+15 \%=37 \%$ dos blogs participam mulheres e em $59+15 \%=$ $74 \%$ dos blogs participam homens. Isso significa que a participação masculina é o dobro da feminina, ou seja, as mulheres participam apenas em pouco mais de um terço dos blogs. A dominância masculina em plataformas de blogs científicos é um fato já verificado em estudos internacionais (MAHRT; PUSCHMANN, 2014), fato este também confirmado para a blogosfera científica brasileira pelo presente estudo.

Esse fenômeno necessita de maior compreensão e clarificação, dado que os blogs científicos são mantidos majoritariamente por estudantes de pós- 
graduação, pós-doutorandos, jornalistas de ciência e cientistas, e a fração de mulheres nessas categorias é considerável - certamente não é apenas um terço da população, com estudos demonstrando que a participação feminina nas atividades científicas no país é crescente: Marques (2012) apontou que dados do Diretório de Grupos de Pesquisa do Conselho Nacional de Desenvolvimento Científico e Tecnológico (CNPq) mostravam que em 2000 a fração de estudantes de doutorado mulheres no país era de 49,1\%, saltando para 55,7\% do total em 2010.

Figura 2 - Distribuição dos blogueiros por gênero.

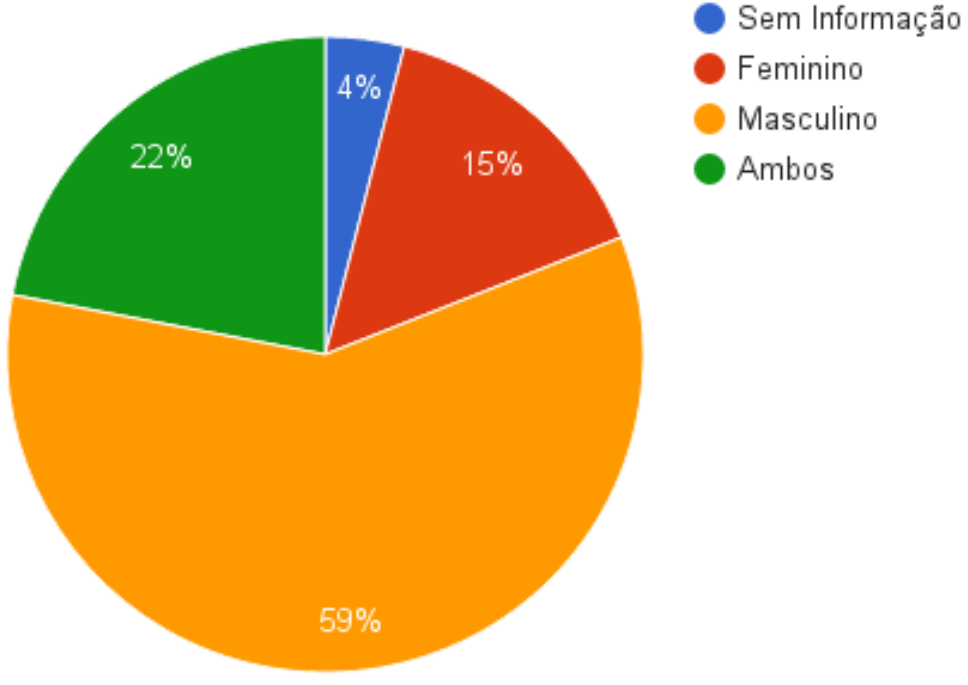

Fonte: Dados da pesquisa.

Quanto à temática (Figura 3) podemos observar uma forte dominância das "Ciências da Vida", também verificada em estudos da blogosfera internacional (FAUSTO et al, 2012). O tema "Ciência Geral" se refere a blogs que comentam ciências biológicas e exatas simultaneamente. Um resultado a ser explicado é o baixo número de blogs dedicados à Matemática e Computação. Isso contrasta, por exemplo, com o grande número de livros de divulgação científica em Matemática e o aparente interesse do público nos mesmos. O pequeno número de blogs dedicados à Química também precisaria ser explicado. Já o baixo número de blogs dedicados à Tecnologia e Inovação incluídos no $\mathrm{ABC}$ pode ser uma falha metodológica, dado que nossa busca por blogs se concentrou na área científica e 
não tecnológica. O número de blogs de ciências humanas pode ter sido afetado pela mesma metodologia.

Figura 3 - Distribuição temática dos blogs brasileiros.

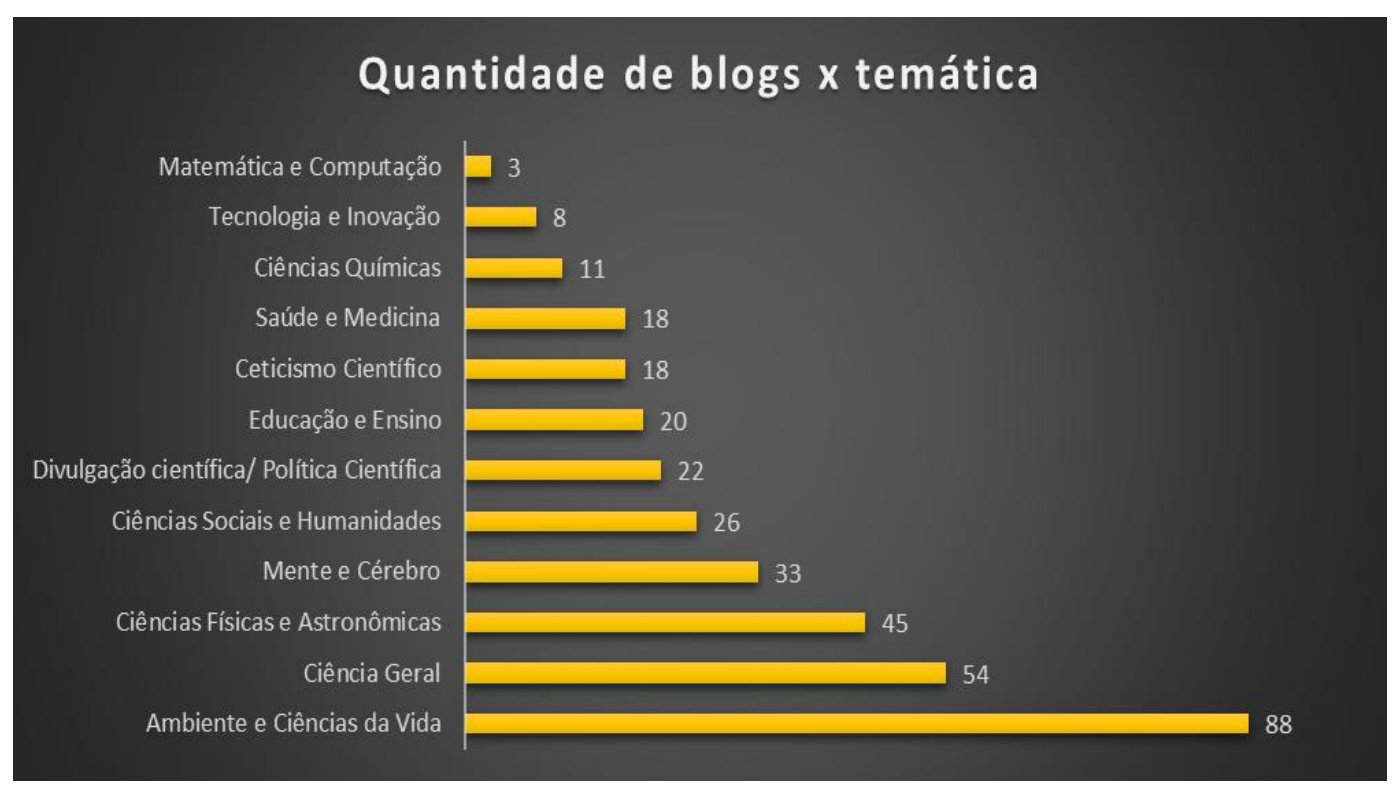

Fonte: Dados da pesquisa.

$\mathrm{Na}$ Figura 4 relatamos a vida média dos $b \log s$, que varia basicamente entre quatro e seis anos. Esse achado é inédito, pois não encontramos estudos correspondentes na literatura internacional e nacional investigando a vida média de blogs científicos. No presente estudo, o resultado mostra que a dispersão em torno desses valores, dada pelo desvio-padrão, é bastante grande, e não podemos dizer a partir da Figura 4 se há correlação entre tempo de vida e temática.

A vida média sobre todos os blogs é de 4,8 anos, com um coeficiente de variação $\mathrm{CV}=0,58$, que é um valor bastante alto. Fica por ser estudado se a vida média dos blogs científicos brasileiros é maior ou menor que a de blogs científicos estrangeiros ou de outras temáticas (política, culturais, turismo, culinária, pessoais, etc.). 
Figura 4 - Vida média dos blogs brasileiros (por temas) e desvio-padrão (barras negras).

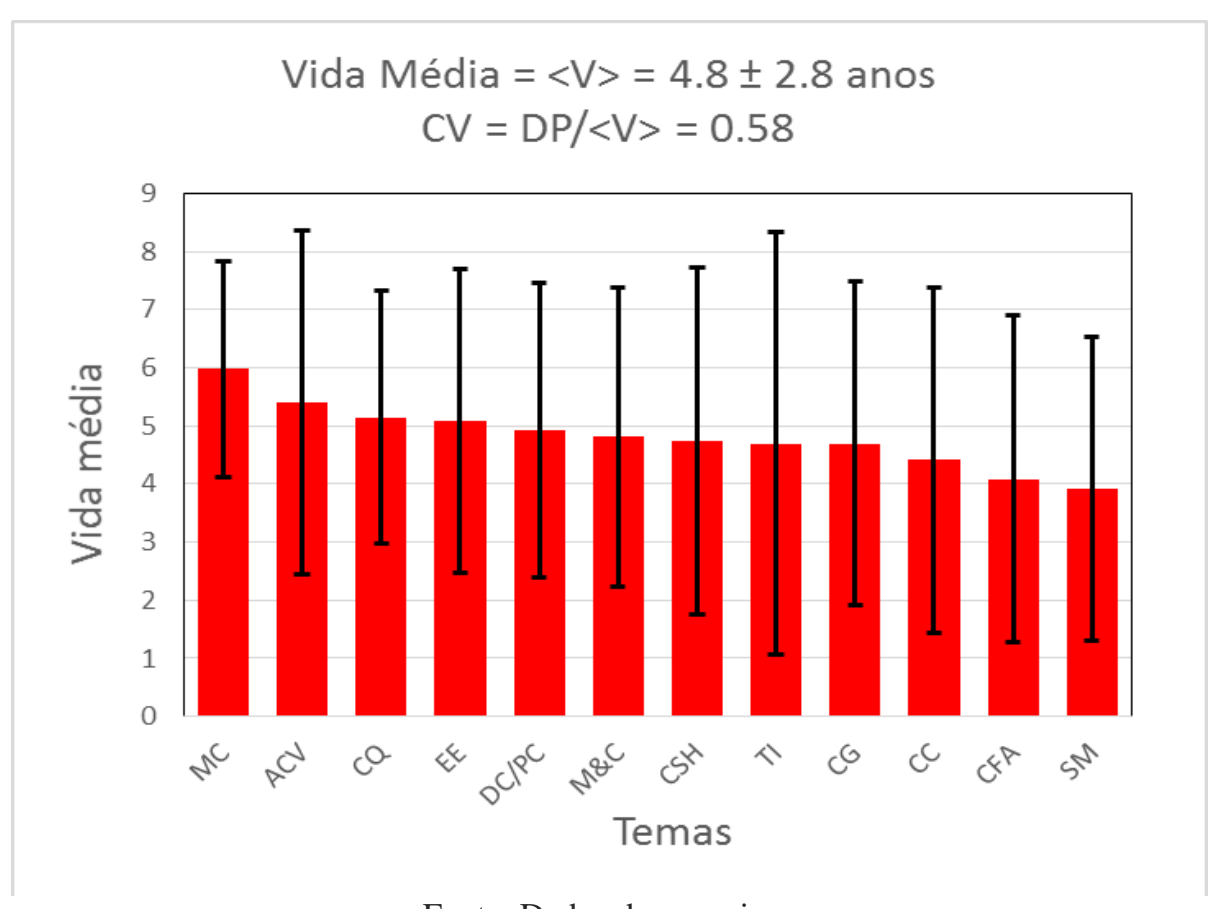

Fonte: Dados da pesquisa.

Na Figura 5 apresentamos a evolução do tempo de atividade dos blogs científicos brasileiros. A curva de primeiro post (em preto) é nossa curva de nascimento $N(t)$ e a curva de último post (em vermelho, no intervalo até $5 / 11 / 2014)$ é nossa curva de morte $M(t)$. Podemos notar um comportamento logístico de $N(t)$, com um crescimento exponencial a partir de 2004, um ponto de inflexão no final de 2008 e uma aparente saturação a partir de 2014. Esse tipo de crescimento logístico é comum em situações em que o crescimento não pode se dar indefinidamente devido à competição por recursos finitos, o que leva à questão sobre o que seriam esses recursos finitos na blogosfera científica: Número de potenciais blogueiros? Tempo disponível do blogueiro? Tempo e atenção dos leitores? Competição com outras mídias sociais?

A curva de último post no período de 5/11/2014 a 5/11/2015 não é considerada uma curva de "morte" (por causa de nossa definição de blog "morto" — ver Metodologia). Nesse último período, vê-se claramente um crescimento rápido do número de últimos posts. Isso se deve simplesmente ao fato de que o blog ainda está "vivo", com últimos posts recentes. 
Figura 5 - Curvas de primeiro post (preto) e último post (vermelho) dos blogs.

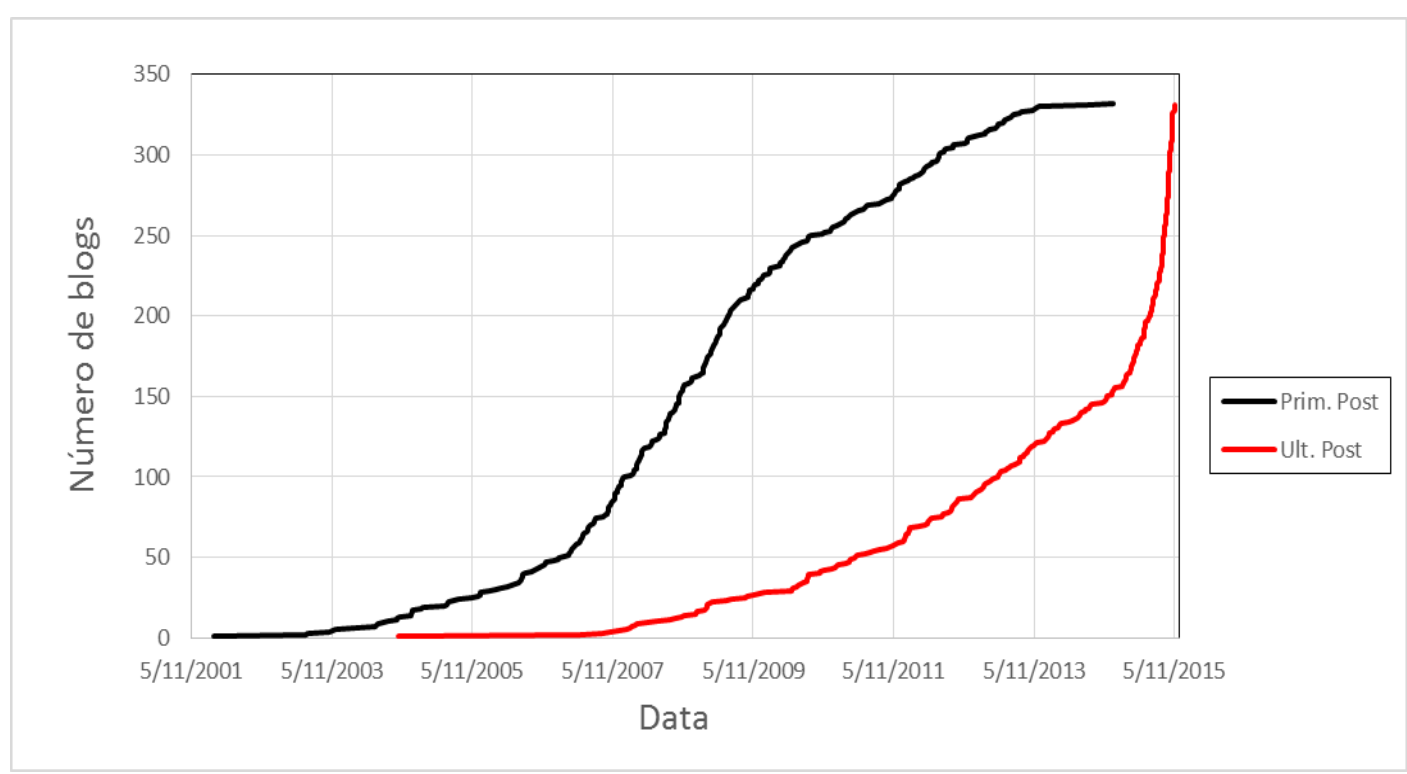

Fonte: Dados da pesquisa.

Na Figura 6 relatamos a curva de blogs ativos $A(t)=N(t)-M(t)$, válida apenas para o intervalo até 5/11/2014. Nela também fica claro o comportamento logístico, ou seja, não apenas o nascimento de blogs $N(t)$ observado na Figura 5, mas o número de blogs ativos $A(t)$ igualmente segue a curva logística.

Figura 6 - Número de blogs ativos em função do tempo $A(t)$.

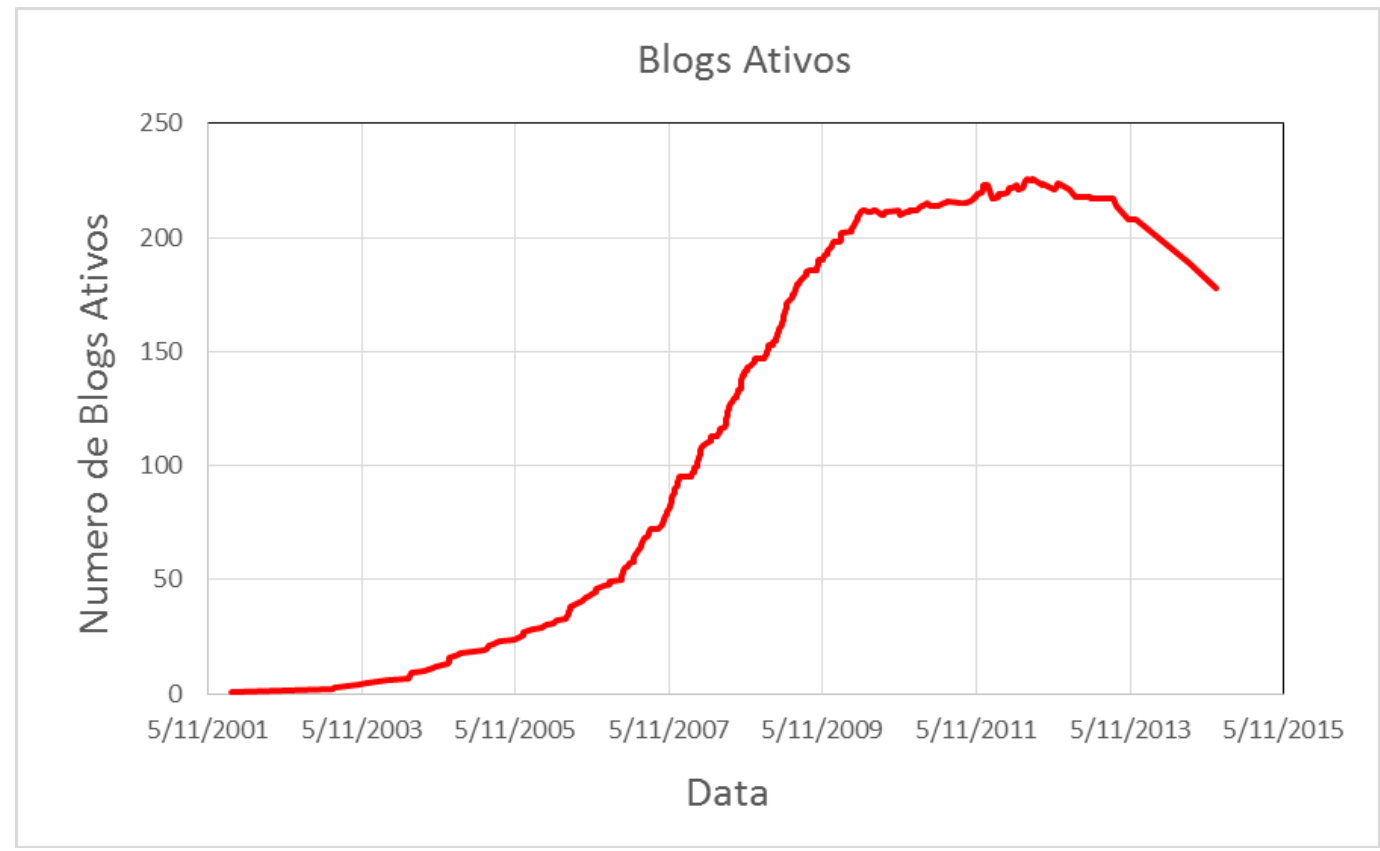

Fonte: Dados da pesquisa. 
Fica também claro o fenômeno de saturação no número de blogs ativos. Acreditamos que essa saturação é real, ou seja, num dado momento, o número de blogs que nascem apenas compensa o número de blogs que morrem. Entretanto, a queda observada no período de 2013 a 2014 precisa ser mais estudada. Ela pode se originar de uma falha metodológica (a princípio, novos blogs são mais difíceis de serem detectados do que blogs antigos) ou, possivelmente, de uma competição com outras mídias sociais (Twitter, Facebook e YouTube). Isso porque divulgadores de ciência passaram a usar o Twitter como plataforma para links para notícias de ciência e começaram a usar o Facebook também como mídia para divulgação científica. Já o YouTube assistiu ao surgimento dos vlogs (video blogs) de ciência. Também surgiram podcasts de divulgação científica.

Ou seja, embora essas mídias não sejam incompatíveis (em geral blogueiros de ciência usam o Twitter e o Facebook para chamar a atenção para o seu blog), certamente envolvem maior gasto de tempo pelo divulgador, a ponto de que o número de postagens em seu blog pode diminuir ou ainda levar à extinção do mesmo.

Outra conjectura, discutida na comunidade de blogueiros de ciência, é a de que a geração inicial que fundou os blogs de ciência de forma entusiástica amadureceu (muitos eram estudantes), de forma que agora se vê comprometida com demandas profissionais e familiares. Aparentemente, uma nova geração de blogueiros de ciência não apareceu com o mesmo entusiasmo, dado que a mesma está mais envolvida com as novas mídias, e o blog, por seu caráter de escrita intensiva, é muitas vezes encarado como mídia trabalhosa e mesmo datada. Todas essas possíveis causalidades do decaimento em volume de postagens percebido na blogosfera científica brasileira e discutidas na comunidade de blogueiros foram documentadas por Takata (2013) e Rodrigues (2014).

Enfatizamos, porém, que essa curiosa queda no número de blogs ativos precisa ser mais bem estudada por ser muito recente, e os dados podem ter problemas metodológicos. Um estudo mais extenso se faz necessário. Por enquanto apenas afirmamos que a queda de atividade na blogosfera científica não é certa, porém é plausível, dada a competição por tempo e o esforço com as novas mídias sociais. 


\section{Conclusões e perspectivas}

Neste trabalho apresentamos estatísticas do estado evolutivo da blogosfera científica brasileira. Cremos que tais estatísticas podem servir como ponto de partida para vários estudos sobre a comunicação científica informal por blogs, incluindo comparativos com a blogosfera científica mundial, principalmente sobre a curva de blogs ativos, verificando se há uma tendência de queda similar e se o fenômeno é global. No presente momento estamos coletando dados sobre a blogosfera científica de Portugal, a fim de realizar tais comparações.

Também a questão da participação feminina precisaria ser mais bem estudada. Finalmente, seria interessante comparar a idade média dos blogs de ciência com seus correspondentes na blogosfera científica internacional, bem como com outros tipos de blogs (política, turismo, pessoais, etc.). Temos a impressão de que os blogs de ciência são mais resilientes e persistentes, mas ainda não temos números concretos para concluir isso. Tais linhas de pesquisa são interessantes e merecem estudos futuros.

\section{Financiamento}

Center for Natural and Artificial Information Processing Systems at the University of São Paulo (CNAIPS-USP).

Centro de Pesquisa, Inovação e Difusão em Neuromatemática - Fundação de Amparo à Pesquisa do Estado de São Paulo (CEPID NeuroMat-Fapesp).

Conselho Nacional de Desenvolvimento Científico e Tecnológico (CNPq).

\section{Referências}

ANEL DE BLOGS CIENTÍFICOS. Sobre. Disponível em: <https://anelciencia.wordpress.com/tag/ciencia/>. Acesso em: 10 set. 1016. 
AQUINO, Maria Clara. Os blogs na Web 2.0: representação e recuperação coletivas de informação. In: AMARAL, Adriana; RECUERO, Raquel;

MONTARDO, Sandra. (Orgs.). Blogs.Com: estudos sobre blogs e comunicação. São Paulo: Momento Editorial, 2009. p. 237-256.

BATTS, Shelley A.; ANTHIS, Nicholas J.; SMITH, Tara. C. Advancing Science through Conversations: Bridging the Gap between Blogs and the Academy. PLoS Biology, San Francisco, v. 6, n. 9, p. 1837- 1841, e240, 2008.

BIK, Holly M.; GOLDSTEIN, Miriam. C. An introduction to social media for scientists. PLoS Biology, San Francisco, v. 11, n. 4, p. 1-8, e1001535, 2013.

BONETTA, Laura. Scientists enter the blogosphere. Cell, Cambridge, v. 129, n. 3, p. 443-445, 2007.

CAREGNATO, Sonia E.; SOUSA, Rodrigo S. C. Blogs científicos.br? Um estudo exploratório. Informação \& Informação, Londrina, v. 15, n. esp., p. 56-75, 2010.

COSTA, Verônica S. Blogs de Ciência: discurso interativo e democratização no campo do jornalismo científico. In: COLÓQUIO INTERNACIONAL TENDÊNCIAS CONTEMPORÂNEAS DA COMUNICAÇÃO CIENTÍFICA: DESAFIOS E PERSPECTIVAS, 1., 2012, Belo Horizonte. Anais... Belo Horizonte: UFMG, 2012.

DENNEN, Vanessa. P. Becoming a blogger: Trajectories, norms, and activities in a community of practice. Computers in Human Behavior, Amsterdam, v. 36, p. 350-358, 2014.

DENNEN, Vanessa P. Constructing academic alter-egos: identity issues in a blogbased community. Identity in the Information Society, Claremont, v. 2, n. 1, p. 23-38, 2009.

DI LUCCIO, Flavia; NICOLACIDA-COSTA, Ana Maria. Blogs: de diários pessoais a comunidades virtuais de escritores/leitores. Psicologia: Ciência e Profissão, Brasília, v. 30, n. 1, p. 132-145, 2010.

FAUSTO, Sibele; TAKATA, Roberto M.; KINOUCHI, Osame. Perfil da Evolução dos Blogs de Ciência Brasileiros. In: COLÓQUIO INTERNACIONAL TENDÊNCIAS CONTEMPORÂNEAS DA COMUNICAÇÃO CIENTÍFICA, 2 ., 2014. Florianópolis. Anais... Florianópolis: UFSC, 2014.

FAUSTO, Sibele et al. Research Blogging: Indexing and Registering the Change in Science 2.0. PLoS ONE, San Francisco, v. 7, n. 12, p. 1-10, e50109, 2012. 
FLORES, Natália F. Entre o protagonismo e a divulgação científica: as estratégias discursivas de constituição do ethos discursivo do cientista blogueiro em blogs de ciência brasileiros. 2016. Tese (Doutorado em Comunicação) Universidade Federal de Pernambuco, Recife, 2016.

FRANÇA, Andressa A. Interação em espaços de divulgação científica na web: uma investigação preliminar no Scienceblogs Brasil. In: SEMINÁRIO DE PESQUISA DA FUNDAÇÃO ESCOLA DE SOCIOLOGIA E POLÍTICA DE SÃO PAULO, 3. 2014, São Paulo. Anais... São Paulo: FESPSP, 2014.

GOMES, Isaltina Maria A. M.; FLORES, Natália M. Um olhar Bakhtiniano sobre os blogs de ciência. Macabéa - Revista Eletrônica do Netlli, Crato, v. 1, n. 2, p. 391-407, 2012.

GUIMARÃES, Cleber. P. Tags: palavras-chave em blogs. In: SIMPÓSIO HIPERTEXTO E TECNOLOGIAS NA EDUCAÇÃO, 2., 2008. Anais... Recife: UFPE, 2008.

GUIMARÃES, Maria. Roda de Ciência. Blog Ciência e Ideias, 3 ago. 2006. Disponível em: <http://cienciaeideias.blogspot.com.br/2006/08/roda-decincia.html>. Acesso em: 10 set. 2016.

JARREAU, Paige B. All the science that is fit to blog: an analysis of science blogging practices. 2015. Tese (Doctor in Philosophy) -The Manship School of Mass Communication, Louisiana State University, Baton Rouge, 2015.

MAHRT, Merja; PUSCHMANN, Cornelius. Science blogging: An exploratory study of motives, styles, and audience reactions. Journal of Science Communication, Trieste, v. 13, p. 1-17, A05, 2014.

MARQUES, Fabrício. Limites da diferença. Pesquisa Fapesp, São Paulo, v. 196, p. 30-31, jun. 2012.

MAURANEN, Anna. Hybridism, edutainment, and doubt: Science blogging finding its feet. Nordic Journal of English Studies, Gothenburg, v. 13, n. 1, p. 7$36,2013$.

OLIVEIRA, Simone M. Os blogs de divulgação científica: informação, notícia, divulgação. 2008. Tese (Doutorado) - Instituto de Estudos da Linguagem, Universidade Estadual de Campinas, Campinas, 2011.

RODRIGUES, Meguie. A crise dos blogs de ciência no Brasil- and le qüiproquó goes on. Blog Medium, 04 set. 2014. Disponível em:

<https://medium.com/@meghier/a-crise-dos-blogs-de-ciencia-no-brasil-and-lequiproquo-goes-on-f6e616d69d94\#.v512zau5a>. Acesso em: 10 set. 2016. 
SCHMIDT, Jan. Blogging Practices: an analytical framework. Journal of Computer Mediated Communication, Hoboken, v. 12, n. 4, p. 1409-1427, 2007.

SHANAHAN, Marie-Claire. Science blogs as boundary layers: creating and understanding new writer and reader interactions through science blogging.

Journalism, Thousand Oaks, v. 12, n. 7, p. 903-919, 2011.

SOUSA, Rodrigo S. C. Trilhas de comunicação científica: links de postagens de pesquisadores brasileiros nos blogs de ciência. 2011. Tese (Doutorado em Comunicação e Informação) - Universidade Federal do Rio Grande do Sul, Porto Alegre, 2011.

TAKATA, Roberto. Há uma crise nos blogues brazucas de ciências? Blog Gene Repórter, 01 out. 2013. Disponível em: <http://genereporter.blogspot.com.br/2013/10/ha-uma-crise-nos-bloguesbrazucas-de.html>. Acesso em: 10 set. 2016.

THELWALL, Mike; KOUSHA, Kayvan. Web indicators for research evaluation. Part 2: social media metrics. El profesional de la información, Barcelona, v. 24, n. 5, p. 607-620, 2015.

WILKINS, John S. The roles, reasons and restrictions of science blogs. Trends in ecology \& evolution, Philadelphia, v. 23, n. 8, p. 411-413, 2008.

\title{
The current state of Brazilian scientific blogosphere
}

\begin{abstract}
This paper investigates quantitatively the Brazilian scientific blogosphere. Sample consists of 346 scientific blogs from the Anel de Blogs Científicos (Scientific Blogs Ring) database, which comprises a large part of the scientific blogs in Brazil, analyzing data collected until November 2015 related to the regional distribution of the blogs, sex of the bloggers, main scientific subject, distribution of blog ages and a growth curve of the active blogs. Results show that most blogs are concentrated in the South and Southeast regions of the country, women participate in $37 \%$ of the blogs and men in $74 \%$ of them, the predominant theme is the Life Sciences and the average life of all blogs is 4.8 years. An important finding is that the number of active blogs seems to have diminished in the last few years. We conjecture that this phenomenon is due to competition with other social media (Facebook, Twitter and vlogs).
\end{abstract}

Keywords: Scientific blogs. Brazilian scientific blogosphere. Social media. Scientific divulgation. 
${ }^{1}$ RESEARCH BLOGGING. Disponível em: <http://researchblogging.org/>.

${ }^{2}$ SCIENCEBLOGS: as the world turns. Disponível em: $\langle\mathrm{http}: / /$ scienceblogs.com/>.

${ }^{3}$ SCIENCESEEKER: science news from science newsmakers. Disponível em: $<$ http://scienceseeker.org/>.

${ }^{4}$ UNIVERSIDADE ESTADUAL DE CAMPINAS. Blogs de Ciência da Universidade Estadual de Campinas. Disponível em: <http://www.blogs.ea2.unicamp.br/pt_BR/>.

${ }^{5}$ SCIENCEBLOGS. ScienceBlogs Brasil: a maior rede de blogs de ciências em português. Disponível em: <http://scienceblogs.com.br/>.

${ }^{6}$ THELWALL, M. Bibliometrics to webometrics. Journal of Information Science, London, v.34, n. 4, 2008.

7 ANEL DE BLOGS CIENTÍFICOS: o estudo da blogosfera cientifica. Disponível em: $<\mathrm{https}: / /$ anelciencia.wordpress.com/>.

Recebido em: 19/09/2016

Aceito em: 08/11/2016 